\title{
Patterns of microsatellite variation through a transition zone of a chromosomal cline in Drosophila americana
}

\author{
MA Schäfer ${ }^{1}$, L Orsini $^{1}$, BF McAllister ${ }^{1,2}$ and C Schlötterer ${ }^{1}$ \\ ${ }^{1}$ Institut für Tierzucht und Genetik, Josef Baumann Gasse 1, A-1210 Wien, Austria; ${ }^{2}$ Department of Biological Sciences, 143 Biology \\ Building, University of Iowa, Iowa City, IA 52242-1324, USA
}

\begin{abstract}
Chromosomal rearrangements have been considered as important barriers to gene flow and were often used in the delineation of species. The original taxonomic designation of Drosophila americana americana and Drosophila americana texana is based on the presence/absence of a centric fusion between the X-and fourth chromosomes. D. a. americana presents the derived fused state, whereas Drosophila a. texana presents the freely segregating ancestral state. The degree of genetic separation between the two chromosomal forms is still controversial, with different genetic markers yielding contrasting results even when the same populations
\end{abstract}

were analyzed. Using 27 polymorphic microsatellites, we re-evaluated patterns of genetic differentiation between six $D$. americana populations sampled through a transition zone of both chromosomal forms in the central United States. Our results clearly reject a scenario of two differentiated species forming a hybrid zone in a region of parapatry and indicate that gene flow minimizes genome-wide differentiation associated with the two chromosomal arrangements.

Heredity (2006) 97, 291-295. doi:10.1038/sj.hdy.6800860; published online 5 July 2006

Keywords: genetic differentiation; gene flow; microsatellites; speciation; geographical separation; hybrid zone

\section{Introduction}

Chromosomal rearrangements, such as reciprocal translocations, inversions or chromosomal fusions, have been considered as important barriers to gene flow through their effect on recombination rather than on hybrid viability (Garagna et al, 1997; Noor et al, 2001; Rieseberg, 2001; Navarro and Barton, 2003). By suppressing recombination in heterozygous individuals, chromosomal rearrangements may act synergistically with isolation genes to diminish gene flow over larger genomic regions than would be otherwise possible and thus may promote population differentiation and even lead to speciation (Rieseberg, 2001; Navarro and Barton, 2003).

Drosophila americana americana and Drosophila americana texana have been the subject of much debate, depending on their classification as two different subspecies within the Drosophila virilis group (Throckmorton, 1982; Powell, 1997) or alternatively as two chromosomal forms of the same species (McAllister, 2002; Vieira et al, 2003). Both forms are native in the central to eastern United States. $D$. a. texana is the southern form, whereas D. a. americana is northerly distributed (Throckmorton, 1982). On the chromosomal level, D. a. americana is characterized by a derived fusion of the $\mathrm{X}$ - and fourth chromosomes, a fusion of Muller's elements A and B, respectively (Muller, 1940; Throckmorton, 1982), whereas D. a. texana retains the

Correspondence: C Schlötterer, Institut für Tierzucht und Genetik, Josef Baumann Gasse 1, A-1210 Wien, Austria.

E-mail: christian.schloetterer@vu-wien.ac.at

Received 13 March 2006; accepted 1 June 2006; published online 5 July 2006 ancestral state, with the $\mathrm{X}$ - and the fourth chromosome segregating independently. Both chromosomal arrangements exhibit a broad geographical transition zone and the relative frequency of the arrangements is tightly associated with latitude. Fused chromosomes are present at high frequency in northern populations, whereas absent or at low frequency in southern populations (Vieira et al, 2001; McAllister, 2002). Apart from the chromosomal fusion, D. a. americana has been characterized by bearing a high frequency in chromosomal inversions that are absent or only present at low frequency in $D$. a. texana (Hsu, 1952). Therefore, recombination between alternative chromosomal forms is likely suppressed throughout a significant proportion of the euchromatic genome. For example, no recombinants were observed in the interval including the centromere and an inversion specific to the fused arrangement of the fourth chromosome (McAllister, 2003). The base of the $X$-chromosome also exhibits signs of reduced recombination (Vieira et al, 2003).

Despite extensive molecular work during the last decade, the degree of genetic separation between the two chromosomal forms remains controversial as different genetic markers yield contrasting results, even when the same populations were analyzed. Sequence data for several nuclear genes located on chromosomes $X, 2,3$, and 4 indicated that flies with and without the $X / 4$ fusion are indistinguishable at the DNA level (Hilton and Hey, 1996; Hilton and Hey, 1997; McAllister, 2002; Vieira et al, 2003), suggesting either high rates of ongoing gene flow or that the species have maintained a large effective population size, which resulted in a large number of shared ancestral alleles. Both hypotheses have been extensively discussed for D. americana (Hilton 
and Hey, 1997; Vieira et al, 2003). More recently, absence of divergence between $D$. a. americana and D. a. texana has also been suggested by a phylogenetic analysis based on the mitochondrial sequences of Cytochrome $b$ and Cytochrome c oxidase subunit II (Caletka and McAllister, 2004). Other studies, however, have detected significant population differentiation. Vieira et al (2001) found that amino-acid replacement polymorphisms at the fused 1 gene, which is located near the base of the X-chromosome close to the fusion breakpoint, are significantly correlated with latitude and longitude in parallel with the chromosomal cline. These observations form the basis for the hypothesis that a balance between gene flow and divergent selection on the karyotypes themselves or on associated genes maintains a clinal distribution for the X/4 fusion (Vieira et al, 2001; McAllister, 2002). However, although this scenario might explain the significant differentiation between the two chromosomal forms at the fused 1 locus as well as at some microsatellite loci mapping to chromosomal element B (Schlötterer, 2000), it cannot account for the genome-wide patterns of differentiation indicated by recent microsatellite analysis. Orsini et al (2004) detected significant genetic differences between populations of D. a. americana and D. a. texana at 11 out of 43 loci analyzed, and these loci were randomly distributed over the genome. These findings not only suggest that differentiation between $D$. a. americana and $D$. a. texana might be more pronounced than previously thought but also indicate that highly polymorphic microsatellites might be more powerful to resolve the genetic relationship between both cytological forms.

Most studies on the differentiation between $D$. $a$. americana and D. a. texana used population samples from the National Drosophila Species Resource Center (currently held at the Tucson Stock Center). These laboratory lines have been maintained in culture for many years and were represented by few strains sampled unevenly from a large geographical range in the United States. This sampling might have affected earlier findings in different manners. For instance, considering the small number of genes and strains analyzed, ancestral polymorphism might account for the lack of divergence between $D$. a. americana and D. a. texana detected in phylogenetic studies (Hilton and Hey, 1996, 1997). Significant differentiation between $D$. a. americana and D. a. texana detected by microsatellite analysis might be attributable to species differentiation, or alternatively to geographic separation as a consequence of uneven sampling (eg about half of the $D$. a. americana lines are derived from western locations in Montana and Nebraska). In this study, we re-evaluate patterns of microsatellite variation between $D$. a. americana and $D$. a. texana by analyzing six populations sampled through a transition zone of both chromosomal forms, in which the species delimitation is not clearly defined. These populations have been collected quite recently and have been used previously in studies of sequence differentiation at six nuclear genes (Vieira et al, 2001, 2003; McAllister, 2002). For the population genetic analysis presented here, we used 27 microsatellites randomly distributed over the genome. This is a significantly larger set of genetic markers than used in many past studies and represents a category of fast evolving DNA markers, which has been shown to be highly informative to infer phylogenetic relationships between closely related species.

\section{Materials and methods}

Fly strains

Flies originated from six localities collected along a broad longitudinal transect through the northern section of the range and a latitudinal transect through the transition zone where putative D. a. americana and D. a. texana populations adjoin in the central United States (Figure 1). The populations furthest apart from each other (OR01 and FP99) are separated by a distance of approximately $1000 \mathrm{~km}$. The exact geographic sampling locations, the number of iso-female lines genetically analyzed as well as the relative frequency of fused-unfused chromosomes for each of the populations studied are given in Table 1. Collections were made in 1996, 1999, and 2001, with the digits in the population ID indicating the year of collection. Methods for examining chromosomal arrangement

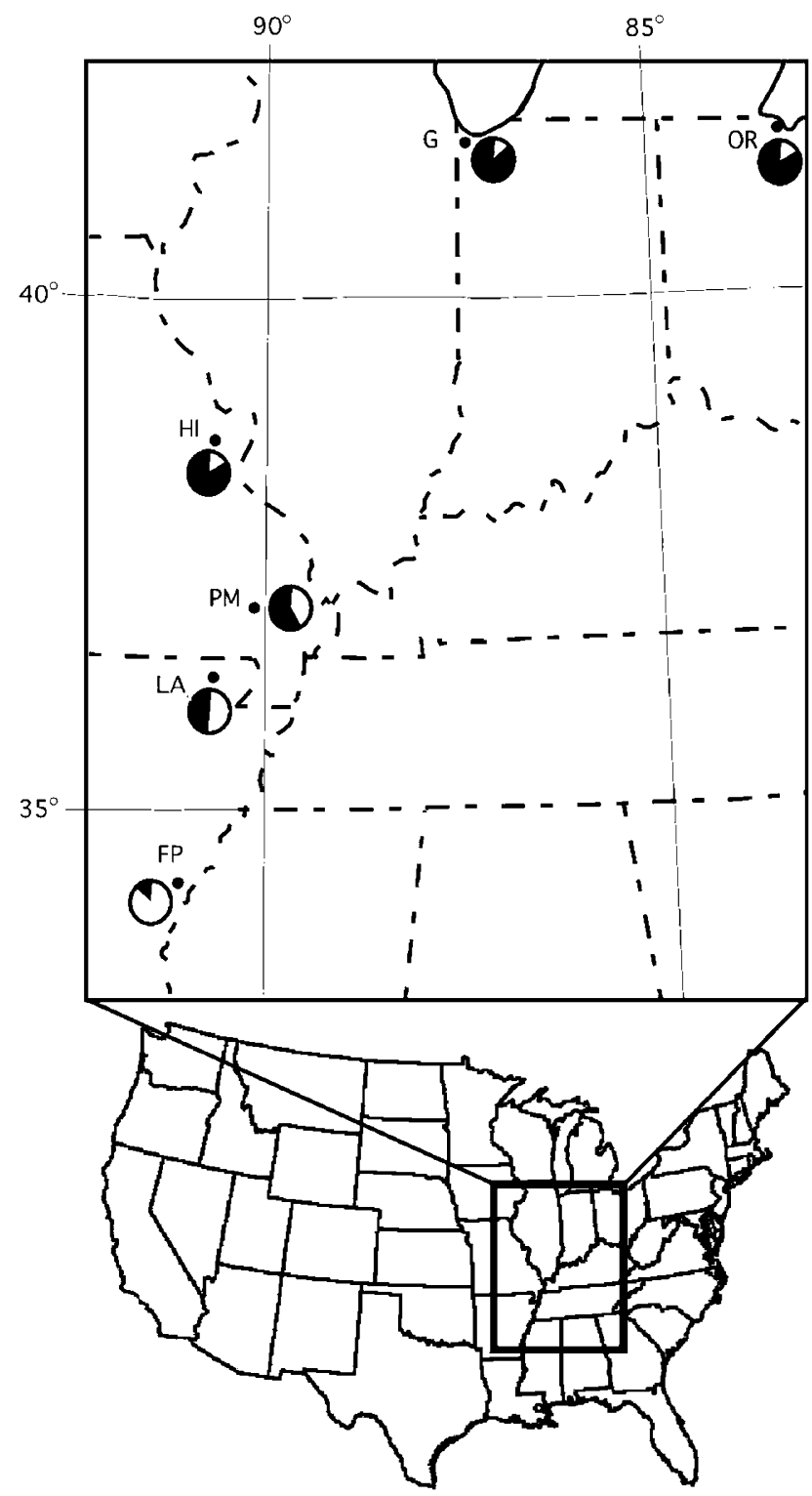

Figure 1 Geographic distribution and frequency of $X / 4$ fusion in samples of D. americana. Each population contains both fused and unfused arrangements, and the black portion of the pie diagrams represents the frequency of the $\mathrm{X} / 4$ fusion. 
Table 1 Populations analyzed with the corresponding number of individuals and the geographic location

\begin{tabular}{|c|c|c|c|}
\hline Population & $\begin{array}{l}\text { No. of } \\
\text { individuals }\end{array}$ & Location & $\begin{array}{l}\text { Chromosomal } \\
\text { state }\end{array}$ \\
\hline OR01 & 18 & $\begin{array}{l}\text { Ottawa NWR Toledo, } \\
\text { Ohio }\end{array}$ & $X / 4$ fusion 85.7 \\
\hline G96 & 11 & Gary, Indiana & $\mathrm{X} / 4$ fusion $98 \%$ \\
\hline HI99 & 15 & Howell Island, Missouri & $\mathrm{X} / 4$ fusion $84.6^{6}$ \\
\hline PM99 & 12 & Puxico, Missouri & $\mathrm{X} / 4$ fusion $55.3 \%$ \\
\hline LA99 & 10 & Lake Ashbaugh, Arkansas & $\mathrm{X} / 4$ fusion $48.7 \%$ \\
\hline FP99 & 19 & Floodgate Park, Arkansas & $\mathrm{X} / 4$ fusion $13.6 \%$ \\
\hline
\end{tabular}

The percentage of $\mathrm{X} / 4$ fusion was determined previously (Vieira et al, 2001; McAllister, 2002) and is available at the website http:// www.biology.uiowa.edu/mcallister/bfm flies.html, edited by B McAllister.

and the frequencies of the alternative forms in these populations have been reported previously (McAllister and Charlesworth, 1999; McAllister, 2001, 2002). Both chromosomal forms are present in all populations. Each line was established from a single wild-caught female, and it has been subsequently maintained in laboratory culture at $18^{\circ} \mathrm{C}$ with transfers about once per month until individuals were processed in ethanol. For each iso-female line, one single female was genotyped.

\section{Markers}

We used a total of 27 polymorphic microsatellite markers to investigate patterns of genetic variation through the transition zone of both chromosomal forms. Most of the microsatellites were originally developed for $D$. virilis (Huttunen and Schlötterer, 2002) and D. montana (Orsini and Schlötterer, 2004) and successfully crossamplified in D. americana. Another set of six markers contained microsatellites previously isolated from P1 clones mapping to the Muller's chromosomal element $\mathrm{B}$ in $D$. americana, which is involved in the $\mathrm{X} / 4$ fusion (Schlötterer, 2000). DNA extraction and PCR amplification followed the protocols given in Schlötterer (2000); Huttunen and Schlötterer (2002); Orsini and Schlötterer (2004).

PCR-products were visualized by autoradiography, and allele sizes were determined running a 'PCR slippage ladder' together with a known size standard adjacent to the samples (Schlötterer and Zangerl, 1999). In order to evaluate the location of microsatellites on different chromosomes, we used a linkage map for D. virilis (Huttunen and Schlötterer, 2002) and a map for D. montana (MA Schäfer et al, unpublished data). Both species are close relatives of $D$. americana and thus should provide a reliable reference for the distribution of the microsatellites studied in the genome of $D$. americana.

\section{Statistical analyses}

We used two complementary statistical approaches to address the genetic separation between the alternative chromosomal arrangements. First, we calculated $F_{\mathrm{ST}}$ between populations (across loci and for each locus separately) using F-statistics according to Weir and Cockerham (1984). Statistical significance of $F_{\mathrm{ST}}$ values was calculated by permuting genotypes among populations for 10000 times. This conservative procedure does not assume Hardy-Weinberg equilibrium and allows for linkage among the loci. The sequential Bonferroni correction procedure was applied to account for multiple testing (Sokal and Rohlf, 1995). All calculations were performed with version 3.12 of the MICROSATELLITEANALYZER software (Dieringer and Schlötterer, 2003). For original MSA input file, see Supplementary information.

Second, we addressed the potential differentiation between the two karyotypes within populations. As the karyotypes of individuals were not known and lines were highly inbred, we did this indirectly using a Bayesian clustering approach to detect cryptic population structure (Pritchard et al, 2000) as implemented in the computer program STRUCTURE version 2.1. The program determines the most probable number of homogeneous groups in a given data using multilocus genotypes and assigns individuals to one or more of them. We assumed prior values of the number of populations, $K$, from 1 to 6 and run 1000000 iterations of the MCMC, after a 'burn-in' period of 100000 iterations. Three independent runs were performed to test the robustness of the results. Long runs were made to assure convergence of the MCMC chain and to obtain accurate estimates. For the simulations, we used a model without a priori consideration of the geographic origin of the samples that allows for admixture between populations.

For all analyses, we randomly selected one allele from each individual per locus to account for the inbreeding effect owing to the propagation of iso-female lines.

\section{Results}

We made two predictions concerning the genetic differentiation between the two karyotypes of $D$. a. americana and $D$. a. texana. If gene flow between individuals of different karyotype is suppressed, we predicted that (i) populations with different frequencies of fused or unfused chromosomes are differentiated from each other and that (ii) flies harboring fused and unfused chromosomes are genetically distinct within populations and are assignable to different groups.

Based on genotypic data of 27 microsatellites, however, we found that overall differentiation between populations was very low, albeit statistically significant (mean $F_{\mathrm{ST}}$ across loci $=0.01 ; 95 \%$ confidence interval from 0.003 to 0.017$)$. Further analysis of pairwise $F_{\mathrm{ST}}$ values between populations (averaged over loci) showed that, whereas four of 20 comparisons were significant, only one remained statistically significant after sequential Bonferroni correction (Table 2). The high genetic similarity between $D$. americana populations appeared to be a genome-wide phenomenon, as none out of the 27 loci studied showed significant $F_{\mathrm{ST}}$ values after sequential Bonferroni correction (Table 3).

We performed a Bayesian analysis of population structure to test for 'cryptic subdivision', which might have remained undetected by the classical $F_{\mathrm{ST}}$ analysis. Previous analysis using representative lines of $D$. a. americana and D. a. texana showed that this Bayesian method has enough statistical power to distinguish between both putative 'species' (Orsini et al, 2004). Contrary to this expectation, however, we failed to recognize distinct genetic entities in our sample: a single cluster was supported with very high probability $(P>0.999)$. 
Table 2 Pairwise $F_{\mathrm{ST}}$ values (Weir and Cockerham, 1984) among D. americana populations (lower triangular matrix) and corresponding $P$-values (upper triangular matrix; ${ }^{a}$ significant after sequential Bonferroni correction)

\begin{tabular}{lcccccc}
\hline Pairwise $F_{S T}$ & OR01 & \multicolumn{1}{c}{ G96 } & HI99 & PM99 & LA99 & FP99 \\
\hline OR01 & - & 0.140 & 0.165 & 0.155 & 0.005 & 0.022 \\
G96 & 0.011 & - & 0.144 & 0.799 & 0.095 & 0.529 \\
HI99 & 0.007 & 0.010 & - & 0.626 & $0.000^{\mathrm{a}}$ & 0.144 \\
PM99 & 0.009 & -0.010 & -0.003 & - & 0.024 & 0.526 \\
LA99 & 0.034 & 0.020 & 0.042 & 0.023 & - & 0.204 \\
FP99 & 0.015 & -0.001 & 0.007 & -0.001 & 0.008 & - \\
\hline
\end{tabular}

Table 3 F-statistics according to Weir and Cockerham (1984) for 27 microsatellite loci across six D. americana populations

\begin{tabular}{lcrc}
\hline Marker & Chromosome & Global $\mathrm{F}_{S T}$ & P-value \\
\hline v11-23 & 2 & 0.018 & 0.096 \\
vir6cs & 2 & 0.027 & 0.144 \\
msat21 & 2 & -0.016 & 0.926 \\
v10-10 & 2 & 0.026 & 0.100 \\
v11-48.2 & 3 & -0.027 & 0.908 \\
msat19 & 3 & 0.021 & $0.047^{*}$ \\
vir12cs & 3 & 0.015 & 0.117 \\
vir34 & 3 & -0.017 & 0.870 \\
vir84 & 3 & -0.004 & 0.596 \\
vir7 & 4 & 0.023 & 0.079 \\
msat11 & 4 & -0.006 & 0.615 \\
mon35 & 4 & 0.028 & 0.095 \\
mon37 & 4 & 0.026 & 0.072 \\
v71.6.1 & $4^{*}$ & 0.023 & 0.110 \\
v68.4 & $4^{*}$ & 0.039 & $0.040^{*}$ \\
v.68.86.1 & $4^{*}$ & 0.016 & 0.150 \\
v68.62 & $4^{*}$ & -0.006 & 0.576 \\
v68.86.2 & $4^{*}$ & 0.035 & 0.124 \\
GPDH & $4^{*}$ & 0.011 & 0.226 \\
Msat4 & 5 & -0.001 & 0.484 \\
v71-03 & $\mathrm{X}$ & -0.006 & 0.494 \\
v68-06.1 & $\mathrm{X}$ & -0.010 & 0.574 \\
msat34 & $\mathrm{X}$ & 0.029 & 0.097 \\
mon26 & $\mathrm{X}$ & -0.018 & 0.884 \\
mon31 & $\mathrm{X}$ & 0.011 & 0.367 \\
mon6 & & 0.026 & 0.089 \\
vir47 & & 0.000 & 0.452 \\
\hline Chrmo & & &
\end{tabular}

Chromosomal location of markers marked with $\left(^{*}\right)$ was determined by chromosome extracted lines (Schlötterer, 2000) and that of the remaining ones from linkage maps of $D$. virilis (Huttunen et al, 2003) and D. montana (MA Schäfer et al, unpublished data).

\section{Discussion}

So far, molecular studies, which have addressed the genetic differentiation between $D$. a. americana and $D$. a. texana, provided ambiguous results leaving the genetic separation unresolved. We re-addressed the genetic differentiation between six $D$. americana populations sampled across a transition zone of the two chromosomal forms using 27 polymorphic microsatellite markers. Our results demonstrate that overall differentiation is low, albeit statistically significant. Nonsignificant differentiation is also supported by a model-based clustering method for multilocus genotype data indicating a single genetic neighborhood over the entire geographical area studied. These findings give insights into the role of recurrent gene flow versus shared ancestral polymorphism, the role of geographical separation versus species separation associated with the $\mathrm{X} / 4$ th chromosomal fusion, and the evolutionary forces causing the chromosomal cline.

Inferring ongoing gene flow from molecular data can be significantly biased by ancestral variation, because both scenarios result in similar patterns of allele sharing. However, our study together with earlier microsatellite studies (Schlötterer, 2000; Orsini et al, 2004) provide strong evidence for ongoing gene flow across the transition zone of both chromosomal arrangements. The significant differentiation reported earlier (Schlötterer, 2000; Orsini et al, 2004) excludes that the low differentiation detected in the present study can be explained by shared ancestral polymorphism alone. Hence, gene flow must be significant. In line with this argument, heterozygous individuals regarding the $X / 4$ fusion show normal fertility (Stone, 1949) and heterozygous individuals for the fusion have been collected in nature (Throckmorton, 1982; McAllister, 2002).

The considerable amount of gene flow through the transition zone further suggests that geographical separation rather than species separation associated with the $\mathrm{X} / 4$ th chromosomal fusion is responsible for the differentiation between D. a. americana and D. a. texana detected earlier. Whereas in previous studies single representative lines from sparsely distributed geographical locations were investigated (Schlötterer, 2000; Orsini et al, 2004), our sampling was confined to the transition zone of the chromosomal cline including much smaller geographical distances among populations and these populations were represented by multiple lines. There was also at least some evidence for geographical separation influencing population differentiation based on the current study. Pairwise $F_{\mathrm{ST}}$ analysis showed four out of 20 comparisons between populations significant, out of which only one remained statistically significant after Bonferroni correction (Table 2). However, population differentiation was not associated with the cline of the $\mathrm{X} / 4$ fusion. These findings support the idea that geographical and not chromosomal separation may be important in shaping the population genetic structure in $D$. americana. It is highly unlikely that the discrepancy among studies results from the use of different microsatellite loci because a significant proportion of the loci were shared between the studies, including loci previously found significantly differentiated (Schlötterer, 2000; Orsini et al, 2004).

The two cytological forms of the X/4th chromosomal fusion in $D$. americana have been frequently regarded as two closely related species forming a hybrid zone in a restricted geographic area of parapatry. Theory on hybrid zones predicts the occurrence of steep allele frequency clines at many loci (Slatkin, 1973; Barton and Hewitt, 1989; Kruuk et al, 1999), which also applies to neutrally evolving DNA markers, such as microsatellites, owing to genetic hitchhiking (Maynard Smith and Haigh, 1974; Slatkin and Wiehe, 1998; Storz, 2005). Although the number of loci and the degree of differentiation may differ dependent on the strength of selection against heterozygous individuals, our data clearly reject the existence of a hybrid zone in a region of parapatry in D. americana. $F_{\mathrm{ST}}$ values estimated for individual loci were not significant, except for two, which is expected by chance alone (Table 3). Thus, the high genetic similarity of $D$. americana populations appears to be a genome-wide phenomenon and not confined to few specific blocks of DNA. 
Furthermore, absence of significant differentiation at many neutral markers demonstrates that the chromosomal cline is unique relative to other segregating variations in the genome and is maintained despite the presence of gene flow. This pattern clearly refutes a scenario in which the chromosomal cline simply reflects neutral rates of dispersal through the region of secondary contact and strongly favors the hypothesis that spatially divergent selection on the karyotypes themselves or on associated genes, for example, the fused 1 locus, maintains the geographical cline of the $X / 4$ fusion in D. americana (Vieira et al, 2001; McAllister, 2002).

In conclusion, although chromosomal fusions/fissions have been reported as barriers to gene flow and have been often used in the delineation of species, the population genetic data presented here provide no evidence for genetic differentiation associated with the $X / 4$ th chromosomal fusion in D. americana. They rather support the idea that $D$. americana exists as a coherent species showing segregating polymorphism for a chromosomal fusion, which may be maintained by divergent selection along an ecological gradient.

\section{Acknowledgements}

We would like to thank Claus Vogl and members of the CS-lab for helpful discussions on earlier versions of the manuscript. This work was funded by the EU Research Training Network (HPRN-CT-2002-00266) and Fonds zur Förderung der wissenschaftlichen Forschung grants to CS and by the National Science Foundation under Grant No. DEB-0420399 to BFM.

\section{References}

Barton NH, Hewitt GM (1989). Analysis of hybrid zones. Annu Rev Ecol Syst 16: 113-148.

Caletka BC, McAllister BF (2004). A genealogical view of chromosomal evolution and species delimitation in the Drosophila virilis species subgroup. Mol Phylogenet Evol 33: 664-670.

Dieringer D, Schlötterer C (2003). Microsatellite analyser (MSA): a platform independent analysis tool for large microsatellite data sets. Mol Ecol Notes 3: 167-169.

Garagna S, Zuccotti M, Redi CA, Capanna E (1997). Trapping speciation. Nature 390: 241-242.

Hilton H, Hey J (1996). DNA sequence variation at the period locus reveals the history of species and speciation events in the Drosophila virilis group. Genetics 144: 1015-1025.

Hilton H, Hey J (1997). A multilocus view of speciation in the Drosophila virilis species group reveals complex histories and taxonomic conflicts. Genet Res 70: 185-194.

Hsu TC (1952). Chromosomal Variation and Evolution in the Virilis Group of Drosophila. University of Texas Publication: Austin, TX.

Huttunen S, Aspi J, Hoikkala A, Schlötterer C (2003). QTL analysis of variation in male courtship song characters in Drosophila virilis. Heredity 92: 263-269.

Huttunen S, Schlötterer C (2002). Isolation and characterization of microsatellites in Drosophila virilis and their cross species amplification in members of the D. virilis. Mol Ecol Notes 2: 593-597.

Kruuk LE, Baird SJ, Gale KS, Barton NH (1999). A comparison of multilocus clines maintained by environmental adaptation or by selection against hybrids. Genetics 153: 1959-1971.

Maynard Smith J, Haigh J (1974). The hitch-hiking effect of a favorable gene. Genet Res 23: 23-35.

McAllister BF (2001). Genetic analysis of sex-chromosome arrangement in Drosophila americana: a laboratory exercise for undergraduate of advanced placement students. Dros Inf. Serv 84: 227-234.

McAllister BF (2002). Chromosomal and allelic variation in Drosophila americana: selective maintenance of a chromosomal cline. Genome 45: 13-21.

McAllister BF (2003). Sequence differentiation associated with an inversion on the neo-X chromosome of Drosophila americana. Genetics 165: 1317-1328.

McAllister BF, Charlesworth B (1999). Reduced sequence variability on the neo-Y chromosome of Drosophila americana americana. Genetics 153: 221-233.

Muller HJ (1940). Bearings of 'Drosophila'work on systematics. In: Huxley J (eds) The New Systematics. Clarendon Press: Oxford. pp 185-268.

Navarro A, Barton NH (2003). Accumulating postzygotic isolation genes in parapatry: a new twist on chromosomal speciation. Evolut Int J Org Evolut 57: 447-459.

Noor MA, Grams KL, Bertucci LA, Reiland J (2001). Chromosomal inversions and the reproductive isolation of species. Proc Natl Acad Sci USA 98: 12084-12088.

Orsini L, Huttunen S, Schlötterer C (2004). A multilocus microsatellite phylogeny of the Drosophila virilis group. Heredity 4: $1-5$.

Orsini L, Schlötterer C (2004). Isolation and characterization of microsatellites in Drosophila montana and their cross-species amplification in D. virilis. Mol Ecol Notes 4: 412-414.

Powell LR (1997). Progress and Prospects in Evolutionary Biology: the Drosophila Model. Oxford University Press: Oxford.

Pritchard JK, Stephens M, Donnelly P (2000). Inference of population structure using multilocus genotype data. Genetics 155: 945-959.

Rieseberg LH (2001). Chromosomal rearrangements and speciation. Trends Ecol Evol 16: 351-358.

Schlötterer C (2000). Microsatellite analysis indicates genetic differentiation of the neo-sex chromosomes in Drosophila americana americana. Heredity 85: 610-616.

Schlötterer C, Zangerl B (1999). The use of imperfect microsatellites for DNA fingerprinting and population genetics. In: Lubjuhn T (eds) DNA Profiling and DNA Fingerprinting. Basel: Birkhäuser. pp 153-165.

Slatkin M (1973). Gene flow and selection in a cline. Genetics 75: 733-756.

Slatkin M, Wiehe T (1998). Genetic hitch-hiking in a subdivided population. Genet Res 71: 155-160.

Sokal RR, Rohlf FJ (1995). Biometry: The Principles and Practice of Statistics in Biological Research, 3rd edn. WH Freeman and company: New York.

Stone WS (1949). The survival of chromosomal variation in evolution. In: Patterson JT (eds) Studies in the Genetics of Drosophila. University of Texas Publication: Austin, TX. pp 18-21.

Storz JF (2005). Using genome scans of DNA polymorphism to infer adaptive population divergence. Mol Ecol 14: 671-688.

Throckmorton LH (1982). The Genetic and Biology of Drosophila. Academy Press: London.

Vieira CP, Coelho PA, Vieira J (2003). Inferences on the evolutionary history of the Drosophila americana polymorphic X/4 fusion from patterns of polymorphism at the X-linked paralytic and elav genes. Genetics 164: 1459-1469.

Vieira J, McAllister BF, Charlesworth B (2001). Evidence for selection at the fused1 locus of Drosophila americana. Genetics 158: 279-290.

Weir BS, Cockerham CC (1984). Estimating F-statistics for the analysis of population structure. Evolut Int J Org Evolut 38: 1358-1370.

Supplementary Information accompanies the paper on Heredity website (http://www.nature.com/hdy) 